\title{
FIXED POINT THEOREM FOR MULTIVALUED MAPPINGS SATISFYING AN IMPLICIT RELATION
}

\author{
DURAN TURKOGLU AND ISHAK ALTUN
}

Abstract. In this paper, we give a common fixed point theorem for multivalued mappings with Hausdorff metric.

\section{Introduction}

Throughout this paper, $X$ stands for a metric space with the metric $d$ whereas $C B(X)$ denotes the family of all nonempty closed bounded subsets of $X$. Let

$$
H(A, B)=\max \left\{\sup _{x \in A} d(x, B), \sup _{y \in B} d(y, A)\right\},
$$

where $A, B \in C B(X)$ and $d(x, A)=\inf \{d(x, y): y \in A\}$. The function $H$ is a metric on $C B(X)$ and is called Hausdorff metric. It is well known that if $X$ is a complete metric space, then so is the metric space $(C B(X), H)$. Let $A, B \in C B(X)$ and $k>1$. In the sequel the following well known fact will be used [4]: for each $a \in A$, there is $b \in B$ such that $d(a, b) \leq k H(A, B)$.

Kaneko and Sessa extend the definition of compatibility to include multivalued mappings in the following way.

Definition 1. ([3]) The mappings $f: X \rightarrow X$ and $S: X \rightarrow C B(X)$ are compatible if $f S x \in$ $C B(X)$ for all $x \in X$ and

$$
\lim _{n \rightarrow \infty} H\left(S f x_{n}, f S x_{n}\right)=0,
$$

whenever $\left\{x_{n}\right\}$ is a sequence in $X$ such that $\lim _{n \rightarrow \infty} S x_{n}=A \in C B(X)$ and $\lim _{n \rightarrow \infty} f x_{n}=t \in A$.

Now, we consider the following conditions.

Condition 1. The mappings $f: X \rightarrow X$ and $S: X \rightarrow C B(X)$ are said to be satisfy Condition 1 iff $f S x \in C B(X)$ for all $x \in X$ and

$$
\lim _{n \rightarrow \infty} H\left(S f x_{n}, f S x_{n}\right) \leq \lim _{n \rightarrow \infty} H\left(S f x_{n}, S x_{n}\right)
$$

whenever $\left\{x_{n}\right\}$ is a sequence in $X$ such that $\lim _{n \rightarrow \infty} S x_{n}=A \in C B(X)$ and $\lim _{n \rightarrow \infty} f x_{n}=t \in A$.

Received September 8, 2005; revised December 12, 2007.

2000 Mathematics Subject Classification. Primary 54H25; Secondary 47H10.

Key words and phrases. Fixed point, implicit relation, multivalued mapping. 
Condition 2. The mappings $f: X \rightarrow X$ and $S: X \rightarrow C B(X)$ are said to be satisfy Condition 2 iff for all $x \in X$ and

$$
\lim _{n \rightarrow \infty} d\left(f f x_{n}, f x_{n}\right) \leq \lim _{n \rightarrow \infty} H\left(S f x_{n}, S x_{n}\right)
$$

whenever $\left\{x_{n}\right\}$ is a sequence in $X$ such that $\lim _{n \rightarrow \infty} S x_{n}=A \in C B(X)$ and $\lim _{n \rightarrow \infty} f x_{n}=t \in A$.

Remark 1. Note that, two compatible maps $f$ and $S$ are satisfy Condition 1.

The following example shows that $f$ and $S$ are satisfy Condition 1 and Condition 2 but they are not compatible.

Example 1. Let $X=[0, \infty)$ be endowed with the Euclidean metric $d$. Let $f x=\frac{x^{2}+2}{2}$ and $S x=\left[x^{2}+1, x^{2}+2\right]$ for each $x \geq 0$. It is clear that $f$ and $S$ are continuous. Let $\left\{x_{n}\right\}$ be a sequence in $X$ such that

$$
\lim _{n \rightarrow \infty} f x_{n}=t, \lim _{n \rightarrow \infty} S x_{n}=A \in C B(X)
$$

then $t \in A$ if and only if $t=1$. Indeed, if $f x_{n} \rightarrow t$, then $t \geq 1$ since $f x=\frac{x^{2}+2}{2} \geq 1$. Again, if $f x_{n} \rightarrow t$, then $x_{n}^{2} \rightarrow 2 t-2$ and so $S x_{n} \rightarrow A=[2 t-1,2 t]$.

Now, if $t \in A$, then $t \in[2 t-1,2 t]$, that is, $2 t-1 \leq t$ and so $t \leq 1$. Now, since $t \geq 1$ and $t \leq 1$, then $t=1$. On the contrary, if $t=1$, then $x_{n} \rightarrow 0$ since $f x_{n} \rightarrow t=1$. Thus $S x_{n} \rightarrow[1,2]=A$ and so $t \in A$. Therefore, we have

$$
0 \neq \lim _{n \rightarrow \infty} H\left(S f x_{n}, f S x_{n}\right)=\frac{1}{2} \leq 1=\lim _{n \rightarrow \infty} H\left(S f x_{n}, S x_{n}\right) .
$$

Thus $f$ and $S$ are satisfy Condition 1 but they are not compatible. On the other hand

$$
\lim _{n \rightarrow \infty} d\left(f f x_{n}, f x_{n}\right)=\frac{1}{2} \leq 1=\lim _{n \rightarrow \infty} H\left(S f x_{n}, S x_{n}\right) .
$$

Thus $f$ and $S$ are satisfy Condition 2 .

\section{Implicit relation}

Implicit relations on metric spaces have been used in many articles (see [2] [5] [6] [8]).

Let $\mathscr{F}$ be the set of all continuous functions $F: R_{+}^{6} \rightarrow R_{+}$satisfying the following conditions:

$F_{1}: F\left(t_{1}, \ldots, t_{6}\right)$ is decreasing in $t_{2}, \ldots, t_{6}$.

$F_{2}$ : there exist an increasing function $f: R_{+} \rightarrow R_{+}, f(0)=0$ and $k>1$ with $f(s)<\frac{s}{k}$ such that the inequality

$\left(F_{a}\right): u \leq k t$ and $F(t, v, v, u, u+v, 0) \leq 0$ or

$\left(F_{b}\right): u \leq k t$ and $F(t, v, u, v, 0, u+v) \leq 0$ implies $t \leq f(v)$.

$F_{3}: F(u, u, u, 0, u, u)>0$ and $F(u, u, 0, u, u, u)>0, \forall u>0$.

Remark 2. Note that, if we choose $f(s)=h s$ with $h k<1$ in $F_{2}$, we obtain implicit relation of Popa [6] 
Example 2. $F\left(t_{1}, \ldots, t_{6}\right)=t_{1}-m \max \left\{t_{2}, t_{3}, t_{4}, \frac{1}{2}\left(t_{5}+t_{6}\right)\right\}$, where $m \in(0,1)$.

$F_{1}$ : Obviously.

$F_{2}$ : Let $u>0, u \leq k t$ and $F(t, v, v, u, u+v, 0)=t-m \max \{u, v\} \leq 0$, where $1<k<\frac{1}{m}$. If $u \geq v$ then $u \leq k t \leq k m u<u$, a contradiction. Thus $u>v$ and $t \leq m v$. Similarly, $u>0, u \leq k t$ and $F(t, v, u, v, 0, u+v) \leq 0$ imply $t \leq m v$. If $u=0$, then $u \leq v$ and $t \leq m v$. Thus $F_{2}$ is satisfying with $f(s)=m s$.

$F_{3}: F(u, u, u, 0, u, u)=F(u, u, 0, u, u, u)=u-m u>0, \forall u>0$.

Example 3. $F\left(t_{1}, \ldots, t_{6}\right)=t_{1}-\phi\left(\max \left\{t_{2}, t_{3}, t_{4}, \frac{1}{2}\left(t_{5}+t_{6}\right)\right\}\right)$, where $\phi: R_{+} \rightarrow R_{+}$increasing, $\phi(0)=0$ and $k>1$ with $\phi(s)<\frac{s}{k}$ for $s>0$.

$F_{1}$ : Obviously.

$F_{2}$ : Let $u>0, u \leq k t$ and $F(t, v, v, u, u+v, 0)=t-\phi(\max \{u, v\}) \leq 0$. If $u \geq v$, then $u \leq k t \leq$ $k \phi(u)<u$, a contradiction. Thus $u<v$ and $t \leq \phi(v)$. Similarly $u>0, u \leq k t$ and $F(t, v, u, v, 0, u+$ $v) \leq 0$ imply $t \leq \phi(v)$. If $u=0$, then $u \leq \phi(v)$. Thus $F_{2}$ is satisfying with $f=\phi$.

$F_{3}: F(u, u, u, 0, u, u)=F(u, u, 0, u, u, u)=u-\phi(u)>0, \forall u>0$.

\section{Main Result}

We need the following lemma for the proof of our main theorem.

Lemma 1. [(7)] For any $t>0, \gamma(t)<t$ if and only if $\lim _{n \rightarrow \infty} \gamma^{n}(t)=0$, where $\gamma^{n}$ denotes the composition of $\gamma n$-times with itself.

Now we give our main theorem.

Theorem 1. Let $(X, d)$ be a complete metric space. Let $f, g: X \rightarrow X$ and $S, T: X \rightarrow C B(X)$ be a continuous mapping such that $f$ and $S$ as well as $g$ and $T$ satisfy Condition 1 and Condition 2. Assume $T(X) \subseteq f(X), S(X) \subseteq g(X)$ and for all $x, y \in X$

$$
F(H(S x, T y), d(f x, g y), d(f x, S x), d(g y, T y), d(f x, T y), d(g y, S x)) \leq 0,
$$

where $F \in \mathscr{F}$. Then $f, g, S$ and $T$ have a common fixed point.

Proof. Let $x_{0}$ be arbitrary point in $X$. We shall construct two sequences $\left\{x_{n}\right\}$ and $\left\{y_{n}\right\}$ of elements in $X$ and sequence $\left\{A_{n}\right\}$ of elements in $C B(X)$. Since $S(X) \subseteq g(X)$, there exists $x_{1} \in X$ such that $y_{1}=g x_{1} \in S x_{0}$. Then there exists an element $y_{2}=f x_{2} \in T x_{1}=A_{1}$, because $T(X) \subseteq f(X)$, such that

$$
d\left(y_{1}, y_{2}\right)=d\left(g x_{1}, f x_{2}\right) \leq k H\left(S x_{0}, T x_{1}\right) .
$$

Since $S(X) \subseteq g(X)$, we may choose $x_{3} \in X$ such that $y_{3}=g x_{3} \in S x_{2}=A_{2}$ and

$$
d\left(y_{2}, y_{3}\right) \leq k H\left(T x_{1}, S x_{2}\right) \text {. }
$$

By induction we produce the sequences $\left\{x_{n}\right\},\left\{y_{n}\right\}$ and $\left\{A_{n}\right\}$ such that

$$
y_{2 n+1}=g x_{2 n+1} \in S x_{2 n}=A_{2 n},
$$




$$
\begin{aligned}
y_{2 n+2} & =f x_{2 n+2} \in T x_{2 n+1}=A_{2 n+1}, \\
d\left(y_{2 n+1}, y_{2 n}\right) & \leq k H\left(S x_{2 n}, T x_{2 n-1}\right), \\
d\left(y_{2 n+1}, y_{2 n+2}\right) & \leq k H\left(S x_{2 n}, T x_{2 n+1}\right)
\end{aligned}
$$

for every $n \in N$. Letting $x=x_{2 n}, y=x_{2 n+1}$ in (3.1), we have successively

$$
\begin{aligned}
& F\left(H\left(S x_{2 n}, T x_{2 n+1}\right), d\left(f x_{2 n}, g x_{2 n+1}\right), d\left(f x_{2 n}, S x_{2 n}\right),\right. \\
& \left.d\left(g x_{2 n+1}, T x_{2 n+1}\right), d\left(f x_{2 n}, T x_{2 n+1}\right), d\left(g x_{2 n+1}, S x_{2 n}\right)\right) \leq 0,
\end{aligned}
$$

and so

$$
\begin{aligned}
& F\left(H\left(S x_{2 n}, T x_{2 n+1}\right), d\left(y_{2 n}, y_{2 n+1}\right), d\left(y_{2 n}, y_{2 n+1}\right),\right. \\
& \left.d\left(y_{2 n+1}, y_{2 n+2}\right), d\left(y_{2 n}, y_{2 n+2}\right), d\left(y_{2 n+1}, y_{2 n+1}\right)\right) \leq 0 .
\end{aligned}
$$

Thus

$$
\begin{aligned}
& F\left(H\left(S x_{2 n}, T x_{2 n+1}\right), d\left(y_{2 n}, y_{2 n+1}\right), d\left(y_{2 n}, y_{2 n+1}\right),\right. \\
& \left.d\left(y_{2 n+1}, y_{2 n+2}\right), d\left(y_{2 n}, y_{2 n+1}\right)+d\left(y_{2 n+1}, y_{2 n+2}\right), 0\right) \leq 0 .
\end{aligned}
$$

From (3.5), (3.6) and $\left(F_{a}\right)$, there exist an increasing function $f: R_{+} \rightarrow R_{+}, f(0)=0$ and $k>1$ with $f(s)<\frac{s}{k}$, we have

$$
H\left(S x_{2 n}, T x_{2 n+1}\right)=H\left(A_{2 n}, A_{2 n+1}\right) \leq f\left(d\left(y_{2 n}, y_{2 n+1}\right)\right)
$$

and so

$$
d\left(y_{2 n+1}, y_{2 n+2}\right) \leq k f\left(d\left(y_{2 n}, y_{2 n+1}\right)\right)
$$

Similarly we obtain

$$
H\left(S x_{2 n}, T x_{2 n-1}\right)=H\left(A_{2 n}, A_{2 n-1}\right) \leq f\left(d\left(y_{2 n-1}, y_{2 n}\right)\right)
$$

and so

$$
d\left(y_{2 n}, y_{2 n+1}\right) \leq k f\left(d\left(y_{2 n-1}, y_{2 n}\right)\right) .
$$

Since $k f(s)<s$ it follows from (3.8), (3.10) and Lemma 1 that $\left\{y_{n}\right\}$ is a Cauchy sequence. Hence there exists $z \in X$ such that $y_{n} \rightarrow z$. Therefore, $g x_{2 n+1} \rightarrow z$ and $f x_{2 n} \rightarrow z$. Also from (3.7) and (3.9) and the fact that $\left\{y_{n}\right\}$ is Cauchy sequence it follows that $\left\{A_{k}\right\}$ is Cauchy sequence in the complete metric space $(C B(X), H)$. Thus $A_{k} \rightarrow A \in C B(X)$. This implies $T x_{2 n+1} \rightarrow A$ and $S x_{2 n} \rightarrow A$ and therefore $z \in A$, because

$$
d(z, A)=\lim _{n \rightarrow \infty} d\left(y_{n}, A\right) \leq \lim _{n \rightarrow \infty} H\left(A_{n-1}, A_{n}\right)=0 .
$$

Since $A$ is closed, $t \in A$ and $f$ and $S$ are satisfying Condition 1 and Condition 2 implies that

$$
\lim _{n \rightarrow \infty} H\left(f S x_{2 n}, S f x_{2 n}\right) \leq \lim _{n \rightarrow \infty} H\left(S x_{2 n}, S f x_{2 n}\right)
$$


and

$$
\lim _{n \rightarrow \infty} d\left(f f x_{2 n}, f x_{2 n}\right) \leq \lim _{n \rightarrow \infty} H\left(S x_{2 n}, S f x_{2 n}\right) .
$$

This along with the continuity of $f$ and $S$ imply that

$$
H(f A, S z) \leq H(A, S z)
$$

and

$$
d(f z, z) \leq H(A, S z)
$$

Now

$$
\begin{aligned}
d(f z, S z) & \leq d\left(f z, f g x_{2 n+1}\right)+d\left(f g x_{2 n+1}, S z\right) \\
& \leq d\left(f z, f g x_{2 n+1}\right)+H\left(f S x_{2 n}, S z\right) \\
& \leq d\left(f z, f g x_{2 n+1}\right)+H\left(f S x_{2 n}, S f x_{2 n}\right)+H\left(S f x_{2 n}, S z\right)
\end{aligned}
$$

and letting $n \rightarrow \infty$ we have

$$
d(f z, S z) \leq \lim _{n \rightarrow \infty} H\left(f S x_{2 n}, S f x_{2 n}\right) \leq H(A, S z) .
$$

Now using (3.1) we have

$$
\begin{aligned}
& F\left(H\left(S z, T x_{2 n+1}\right), d\left(f z, g x_{2 n+1}\right), d(f z, S z),\right. \\
& \left.d\left(g x_{2 n+1}, T x_{2 n+1}\right), d\left(f z, T x_{2 n+1}\right), d\left(g x_{2 n+1}, S z\right)\right) \leq 0,
\end{aligned}
$$

and

$$
\begin{aligned}
& F\left(H\left(S z, T x_{2 n+1}\right), d\left(f z, g x_{2 n+1}\right), d(f z, S z),\right. \\
& \left.d\left(g x_{2 n+1}, T x_{2 n+1}\right), d\left(f z, f x_{2 n+2}\right)+d\left(f x_{2 n+2}, T x_{2 n+1}\right), d\left(g x_{2 n+1}, S z\right)\right) \leq 0 .
\end{aligned}
$$

Letting $n \rightarrow \infty$ we obtain

$$
F(H(S z, A), d(f z, z), d(f z, S z), d(z, A), d(f z, z)+d(z, A), d(z, S z)) \leq 0
$$

and so

$$
F(H(S z, A), H(S z, A), H(S z, A), 0, H(S z, A), H(S z, A)) \leq 0
$$

which is a contradiction to $F_{3}$. Thus $H(S z, A)=0$ and so, from (3.11) and (3.12) we have $z=$ $f z \in S z$. Similarly we have $z=g z \in T z$. Thus $z$ is a common fixed point of this four mappings.

Corollary 1. Let $(X, d)$ be a complete metric space. Let $f, g: X \rightarrow X$ and $S, T: X \rightarrow C B(X)$ be a continuous mapping such that $f$ and $S$ as well as $g$ and $T$ satisfy Condition 1 and Condition 2. Assume $T(X) \subseteq f(X), S(X) \subseteq g(X)$ and that $H(S x, T y) \leq r d(f x, g y)$ for all $x, y \in X$ where $0<r<1$. Then $f, g, S$ and $T$ have a common fixed point.

Example 4. Let $X=[0,1]$ be endowed with the Euclidean metric $d$. Let $f x=\frac{x}{2}, g x=\frac{x^{2}}{3}$, $S x=\left[0, \frac{x}{4}\right]$ and $T x=\left[0, \frac{x^{2}}{6}\right]$. It is clear that these mappings are continuous and $S(X)=\left[0, \frac{1}{4}\right] \subset$ 
$\left[0, \frac{1}{2}\right]=f(X)$ and $T(X)=\left[0, \frac{1}{6}\right] \subset\left[0, \frac{1}{3}\right]=g(X)$. Also $f$ and $S$ as well as $g$ and $T$ are satisfy Condition 1 and Condition 2. Indeed, let $\left\{x_{n}\right\}$ be a sequence in $X$ such that

$$
\lim _{n \rightarrow \infty} f x_{n}=t, \lim _{n \rightarrow \infty} S x_{n}=A \in C B(X),
$$

then $t \in A$ if and only if $t=0$. Therefore,

$$
\lim _{n \rightarrow \infty} H\left(S f x_{n}, f S x_{n}\right)=\lim _{n \rightarrow \infty} d\left(f f x_{n}, f x_{n}\right)=0=\lim _{n \rightarrow \infty} H\left(S f x_{n}, S x_{n}\right) .
$$

Thus $f$ and $S$ are satisfy Condition 1 and Condition 2. Again, for all $x, y \in X$, we have

$$
\begin{aligned}
H(S x, T y) & =H\left(\left[0, \frac{x}{4}\right],\left[0, \frac{y^{2}}{6}\right]\right) \\
& =\left|\frac{x}{4}-\frac{y^{2}}{6}\right| \\
& =\frac{1}{2}\left|\frac{x}{2}-\frac{y^{2}}{3}\right| \\
& =\frac{1}{2} d(f x, g y) .
\end{aligned}
$$

Consequently, these mappings are satisfy all conditions of Corollary 1, then they have a common fixed point in $X$.

Theorem 2. [(1)] Let $(X, d)$ be a complete metric space. Let $f, g: X \rightarrow X$ and $S, T: X \rightarrow$ $C B(X)$ be a continuous mapping such that $f$ is compatible with $S$ and $g$ is compatible with T. Assume $T(X) \subseteq f(X), S(X) \subseteq g(X)$ and that $H(S x, T y) \leq r d(f x, g y)$ for all $x, y \in X$ where $0<r<1$. Then there is a coincidence point for $f$ and $S$, as $g$ and $T$.

Remark 3. In Theorem 2, Azam and Beg [1] find a coincidence point of mappings assumed compatibility. In Corollary 1 , we find a common fixed point of mappings with Condition 1 and Condition 2 replaced by compatibility.

\section{Acknowledgement}

The authors are thankful to the referees for their valuable comments in modifying the first version of this paper.

\section{References}

[1] A. Azam and I. Beg, Coincidence points of compatible multivalued mappings, Demonstratio Math. 29(1996), 17-22.

[2] M. Imdad, S. Kumar and M. S. Khan, Remarks on some fixed point theorems satisfying implicit relations, Rad. Math. 11(2002), 135-143.

[3] H. Kaneko and S. Sessa, Fixed point theorems for compatible multivalued and single-valued mappings, Internat. J. Math. and Math. Sci. 12(1989), 257-262. 
[4] S. B. Nadler, Multivalued contraction mappings, Pacific J. Math. 20(1969), 457-488.

[5] V. Popa, Some fixed point theorems for compatible mappings satisfying an implicit relation, Demonsratio Math. 32(1999), 157-163.

[6] V. Popa, A general coincidence theorem for compatible multivalued mappings satisfying an implicit relation, Demonstratio Math. 33(2000), 159-164.

[7] S. P. Singh and B. A. Meade, A common fixed point theorem, Bull. Austral. Math. Soc. 16(1977), 4953.

[8] S. Sharma and B. Desphande, On compatible mappings satisfying an implicit relation in common fixed point consideration, Tamkang J. Math. 33(2002), 245-252.

Department of Mathematics, Faculty of Science and Arts, Gazi University, 06500 Teknikokullar, Ankara, Turkey.

E-mail: dturkoglu@gazi.edu.tr

Department of Mathematics, Faculty of Science and Arts, Kirikkale University, 71450 Yahsihan, Kirikkale, Turkey.

E-mail:ialtun@kku.edu.tr, :ishakaltun@yahoo.com 